\title{
Analytical Relation Between the Concentration of Species at the Electrode Surface and Current for Quasi-Reversible Reactions
}

\author{
S. Pavithra ${ }^{1}$, L. Rajendran ${ }^{1, *}$, Sunil Kumar ${ }^{2}$ \\ ${ }^{1}$ Department of Mathematics, SethuInistitute of Technology, Kariapatti, India \\ ${ }^{2}$ Department of Mathematics, National Institute of Technology, Jamshedpur, India
}

Email address:

pavithrasivakumar87@gmail.com (S.Pavithra),raj_sms@rediffmail.com (L. Rajendran), skumar.math@nitjsr.ac.in (S. Kumar)

*Corresponding author

\section{To cite this article:}

S. Pavithra, L. Rajendran, Sunil Kumar. Analytical Relation Between the Concentration of Species at the Electrode Surface and Current for Quasi-Reversible Reactions. Applied and Computational Mathematics. Vol. 6, No. 2, 2017, pp. 83-87. doi: 10.11648/j.acm.20170602.13

Received: February 23, 2017; Accepted: March 14, 2017; Published: March 27, 2017

\begin{abstract}
Cyclic voltammetry take into account the mass transport of species and the kinetics at the electrode surface. Analytical solutions of these models are not well-known due to the complexity of the boundary conditions. In this study we present analytical relation between the concentration at the electrode surface and the current for quasi-reversible reaction.A new semi analytic description ofquasi-reversible cyclic voltammetry at a electrode is obtained, assuming equal diffusion coefficients. It provides rigorous and complete expression for the voltamettric current, in the form of the integral or the integral equation. This solution method can be extended to cases that are more general and may be useful for benchmarking purposes.
\end{abstract}

Keywords: Mathematical Modeling, Boundary Value Problems, Non Linear Equations, Quasi-Reversible

\section{Introduction}

Cyclic voltammetry is one of the main tools used to characterize electrochemical systems and has a broad range of applications. This method is frequently used for analyzing electroactive species and surfaces and for the determination of reaction mechanisms and rate constants. Investigators over the years have sought analytical expressions and relationships to enable a theoretical interpretation and understanding of experimentally recorded cyclic voltammograms and other electrochemical characterization techniques [1-6]. Bortels et al. [7] found analytical solutions for the one dimensional steady state transport of ions in an electrolyte between two planar electrodes. Molina et al. [8] derived analytical expressions for the current or potential response and concentration profiles for the reversible ion transfer at the interface between two immiscible electrolyte solutions.

Berzins and Delahay [9] derived an equation for oscillographicpolarographic waves corresponding to the reversible deposition of an insoluble substance and compared their results with experimental data. White and Lawson [4] presented a solutions for the voltammetric deposition and dissolution of a metal from an electrode accounting for spherical effects as well as kinetics, uncompensated cell resistance, and submonolayer metal deposition. Lantelme and Cherrat [3] explored the analytical solutions to the cyclic voltammetry process.Oldham and Myland [10] derived mathematical solutions for the cyclic voltammetry of strong adsorption such that the redox pair is confined to the surface.In addition, Oldham and Myland [11], developed a semi-analytical method for describing the current in cyclic voltammetry.

Eswari and Rajendran [12] reported the closed-form analytical expressions for the concentration in an EC reaction for limiting cases of small and large reaction rates at the electrode and small and large time intervals. In that investigation, the authors employed the equation in planar geometry to describe the mass transport and the ButlerVolmer equation at the electrode surface to account for the kinetics. The asymptotic expressions for the normalized current were derived by using the Laplace transformation.

Furthermore, Eswari and Rajendran [13] explored analytical solutions for the $\mathrm{EC}_{2}$ cyclic voltammetry model 
[13] using He's homotopy perturbation method.Samin, and Jinsuo Zhang [14] derive analytical solutions to the planar cyclic voltammetry model which describes mass transport of species through the equations and the kinetics at the electrode surface through the Nernst and Butler-Volmer equations. In this study we have obtained the relation between the concentration ferrocyanideat the electrode surface and the current for quasi-reversible reaction.

Table 1. Nomenclature.

\begin{tabular}{ll}
\hline Symbols & Definitions \\
\hline$c_{r e d}$ & The concentration of redox species \\
$c_{o x}$ & The concentration of oxidised species \\
$D_{r e d}$ & Diffusion coefficient of redox species \\
$D_{o x}$ & Diffusion coefficient of oxidised species \\
$k^{e}$ & EC rate constant \\
$c_{r e d}(0, t), c_{o x}(0, t)$ & The concentrations of the reacting species at \\
$c_{r e d, \infty}, c_{o x, \infty}$ & the electrode surface \\
$E$ & The concentrations of the reacting species in \\
$E_{r}$ & the bulk of the solution \\
$\beta$ & Electrode potential \\
$F$ & The equilibrium electrode potential \\
$R$ & symmetry factor \\
$T$ & Faraday constant \\
$a=C_{r e d} / C_{r e d}$ & The universal gas constant \\
$b=C_{o x} / \frac{C_{o x}}{C_{r e d}}$ & The thermodynamic temperature \\
$\tau=t F v / R T$ & Dimensionlessconcentration of redox species \\
$X=Z \sqrt{F V / R T D_{r e d}}$ & Dimensionlessconcentration of oxidised \\
$\xi=\frac{K e}{D_{r e d}} \sqrt{R T D_{r e d} / F V}$ & Dimensionless distance \\
\hline & Dimensionless parameter \\
&
\end{tabular}

$$
r(t)=k^{e}\left\{\frac{c_{r e d}(0, t)}{c_{r e d, \infty}} \exp \left(\beta F\left(E(t)-E_{r}\right) / R T\right)-\frac{c_{o x}(0, t)}{c_{o x, \infty}} \exp \left(-(1-\beta) F\left(E(t)-E_{r}\right) / R T\right)\right\}
$$

Where $k^{e}$ is the EC rate constant, $c_{\text {red }}(0, t), c_{o x}(0, t), c_{r e d, \infty}, c_{o x, \infty}$ are the concentrations of the reacting species at the electrode surface and in the bulk of the solution, respectively. $E$ is the electrode potential, $E_{r}$ is the equilibrium electrode potential, and $\beta$ is a symmetry factor, whereas $f=F / R T$, where $F$ is the Faraday constant, $R$ is the universal gas constant, and $T$ is the thermodynamic temperature. The EC constant $k^{e}$ is defined as follows:

$$
K^{e}=K_{r e d}^{*} c_{r e d, \infty}^{*} \exp \left(\beta f\left(E_{r}\right)\right)=K_{o x}^{*} C_{o x, \infty}^{*} \exp \left(-(1-\beta) f\left(E_{r}\right)\right)
$$

We introducethe following set of dimensionless variables:

$$
\begin{aligned}
& a=\frac{C_{r e d}}{C_{r e d^{*}}}, b=\frac{C_{o x}}{C_{r e d^{*}}}, \quad \tau=\frac{t F v}{R T}, \\
& X=Z \sqrt{\frac{F V}{R T D_{r e d}}}, \xi=\frac{K e}{D_{r e d}} \sqrt{\frac{R T D_{r e d}}{F V}}
\end{aligned}
$$

\section{Mathematical Formulation of the Problem}

Consider the EC reaction

$$
\left[\mathrm{Fe}(\mathrm{CN})_{6}\right]^{4-} \leftrightarrow\left[\mathrm{Fe}(\mathrm{CN})_{6}\right]^{3-}+e^{-}
$$

The concentration profiles of the red and ox forms can beobtained by solving the diffusion equation, which can be simplified in the case of a rotating disk electrode (RDE) to the following one dimensional form.

$$
\begin{gathered}
\frac{\partial c_{r e d}(z, t)}{\partial t}=D_{r e d} \frac{\partial^{2} c_{r e d}(z, t)}{\partial z^{2}} \\
\frac{\partial c_{o x}(z, t)}{\partial t}=D_{r e d} \frac{\partial^{2} c_{o x}(z, t)}{\partial z^{2}}
\end{gathered}
$$

Where $c_{r e d}, c_{o x}$ are the concentration of redox and oxidisedspecies. $D_{\text {red }}$ and $D_{o x}$ are the corresponding diffusion coefficient.The initial and boundary conditions for the aboveequationsaregiven by [15].

$$
\begin{aligned}
& \mathrm{c}_{\text {red }}(\mathrm{z}, 0)=\mathrm{c}_{r e d}^{*}, \quad \mathrm{c}_{o x}(\mathrm{z}, 0)=\mathrm{c}_{o x}^{*}, \quad \text { when } t=0 \\
& \left.D_{i} \frac{\partial c_{i}(z, t)}{\partial z}\right|_{z=0}=\frac{i(t)}{n F A}= \pm r(t) \text { where }+ \text { or }- \text { sign stands for } \\
& \mathrm{i}=\text { red } \text { or } \text { ox } \\
& c_{i}\left(\delta_{\mathrm{D}, \mathrm{i}}, \mathrm{t}\right)=c_{\infty} \quad \text { where } \quad \delta_{\mathrm{D}, i}=1.61 D_{i}^{1 / 3} v^{1 / 6} \omega_{r}^{-1 / 2}
\end{aligned}
$$

In the case of Butler- Vomer kineticsreaction rater $(\mathrm{t})$ is given by

Using the above dimensionless variables the diffusion equations (1) and (2) are expressed in the following dimensionless form:

$$
\begin{aligned}
& \frac{\partial a}{\partial \tau}=\frac{\partial^{2} a}{\partial X^{2}} \\
& \frac{\partial b}{\partial \tau}=\xi \frac{\partial^{2} b}{\partial X^{2}}
\end{aligned}
$$

The dimensionless initial and boundary conditions are

$$
a=1, \quad b=0 \quad \text { when } \tau=0 \text {; }
$$

$$
a=\frac{C_{\infty}}{C_{r e d^{*}}}, \quad b=\frac{C_{\infty}}{C_{r e d^{*}}} \quad \text { when } X=\delta_{D, i} \sqrt{\frac{F V}{R T}}=\gamma
$$




$$
\begin{aligned}
& \left.\frac{d a}{d X}\right|_{X=0}=K_{1} \exp \left[l_{1}+\beta \tau\right) a(0, \tau)-K_{2} \exp \left[l_{2}+\alpha \tau\right) b(0, \tau) \\
& \left.\frac{d b}{d X}\right|_{X=0}=-K_{3} \exp \left[l_{1}+\beta \tau\right) a(0, \tau)-K_{4} \exp \left[l_{2}+\alpha \tau\right) b(0, \tau)
\end{aligned}
$$

Where

$$
\alpha=1-\beta, \quad l_{1}=\frac{\beta F\left(E_{1}-E_{r}\right)}{R T},
$$

$$
\begin{gathered}
l_{21}=\frac{(\beta-1) F\left(E_{1}-E_{r}\right)}{R T}, \quad K_{1}=\frac{k_{e} R T}{C_{r e d, \infty} D_{r e d} F V} \\
K_{2}=\frac{k_{e} R T}{C_{o x, \infty} D_{r e d} F V} \quad K_{3}=\frac{k_{e} R T}{C_{r e d, \infty} D_{o x} F V} \\
K_{4}=\frac{k_{e} R T}{C_{o x, \infty} D_{o x} F V}
\end{gathered}
$$

We get the following relation between the surface concentration and current (Appendix A)

$$
a(0, \tau)=1-\frac{1}{m \sqrt{\pi}}\left[\int_{0}^{\tau} \frac{i(\tau)}{\sqrt{t-\tau}}\left[1-2 \exp \left(-\gamma^{2} / 4(t-\tau)\right] d \tau\right)\right]
$$

Similarly we get

$$
\begin{gathered}
C_{r e d}(0, \tau)=C_{r e d}{ }^{*}-\left\{\left[1 /\left(\sqrt{D_{r e d}} n F R A \sqrt{F V / R T}\right)\right]\left\{\int_{0}^{\tau} \frac{i(\tau)}{\sqrt{t-\tau}}\left[1-2 \exp \left(-\gamma^{2} / 4(t-\tau)\right] d \tau\right)\right\}\right\} \\
C_{o x}(0, \tau)=\left[1 /\left(\sqrt{D_{r e d}} n F R A \sqrt{F V / R T}\right)\right]\left[\int_{0}^{\tau} \frac{i(\tau)}{\sqrt{t-\tau}}\left[1-2 \exp \left(-\gamma^{2} / 4(t-\tau)\right] d \tau\right)\right]
\end{gathered}
$$

The above equation can be rewritten as

$$
\begin{array}{r}
C_{r e d}(0, \tau)=C_{r e d}^{*}-\frac{I(\tau)}{\sqrt{D_{r e d}} n F R A \sqrt{F V / R T}} \\
C_{o x}(0, \tau)=\frac{I(\tau)}{\sqrt{D_{o x}} n F R A \sqrt{F V / R T}}
\end{array}
$$

Under few diffusion control condition $C_{r e d}(0, \tau)=0$ and therefore $I(t)$ reaches the limit value $I_{L}$

$$
C_{\text {red }}(0, \tau)=\frac{I_{L}-I(\tau)}{\sqrt{D_{\text {red }}} n F R A \sqrt{F V / R T}}
$$

Where

$$
\begin{aligned}
& I(\tau)=\left\{\int_{0}^{\tau} \frac{i(\tau)}{\sqrt{t-\tau}}\left(1-2 e^{-\gamma^{2} / 4(t-\tau)} d \tau\right)\right\} \text { and } \\
& I_{L}=C_{\text {red }}^{*} \sqrt{D_{\text {red }}} n F R A \sqrt{F V / R T}
\end{aligned}
$$

\section{Conclusions}

We have derived the analytical relation between the cocentration of the species at the electrode surface and currentfor quasi-reversible reactions. From this result we can also obtain the concentration ofof the species at the electrode surface and currentfor reversible reactions. The extension of the procedure to other convection difussion process in rotating disc, rotating ring-disc electrodes,etc apart from the stydy of nonlinear convection difussion process in all hydronynamicelectrodes seems possible.

\section{Acknowledgement}

The authors are very much grateful to the referees for the valuable suggestions. This work was supported by the DST SERB/EMR/2015/002279, New Delhi, India.The authors are thankful to Mr. S. Mohamed Jaleel, The Chairman, Dr. A. Senthilkumar, The Principal, and Dr. P. G. Jansi Rani, Head of the Department of Mathematics, SethuInistitute of Technology, Kariapatti-626115, Tamilnadu, India for their encouragement.

Appendix A: Analytical relation between the surface concentration and the current.

The dimensionless form of one dimensional plannar diffusion are described by the following equations: 


$$
\begin{array}{r}
\frac{\partial a}{\partial \tau}=\frac{\partial^{2} a}{\partial X^{2}} \\
\frac{\partial b}{\partial \tau}=\xi \frac{\partial^{2} b}{\partial X^{2}}
\end{array}
$$

The initial and boundary conditions are

$$
\begin{gathered}
a=1, \quad b=0 \quad \text { when } \tau=0 \\
a=\frac{C_{\infty}}{C_{r e d^{*}}}, \quad b=\frac{C_{\infty}}{C_{r e d^{*}}} \quad \text { when } X=\delta_{D} \sqrt{\frac{F V}{R T}} \\
\left.\frac{d a}{d X}\right|_{X=0}=K_{1} \exp \left[l_{1}+\beta \tau\right) a(0, \tau)-K_{2} \exp \left[l_{2}+\alpha \tau\right) b(0, \tau) \\
\left.\frac{d b}{d X}\right|_{x=0}=-K_{3} \exp \left[l_{1}+\beta \tau\right) a(0, \tau)-K_{4} \exp \left[l_{2}+\alpha \tau\right) b(0, \tau)
\end{gathered}
$$

Taking the Laplace transform of Eqn. (A1), we get

$$
\bar{a}(X, s)=c_{1} e^{\sqrt{s X}}+c_{2} e^{-\sqrt{s X}}+\frac{1}{s}
$$

Using the boundary conditionEqns. (A3)-(A6) we get

$$
\bar{a}(0, s)=c_{1}\left[2-e^{\sqrt{s \gamma}}\right]+\frac{1}{s}
$$

Now current becomes

$$
\frac{i(\tau)}{n F A}=\left.D_{\text {red }} c_{r e d^{*}} \sqrt{\frac{F V}{R T D_{\text {red }}}} \frac{d a(X, s)}{d X}\right|_{X=0}
$$

Taking Laplaceonboth sides

$$
\frac{i(s)}{n F A}=\left.D_{r_{e d}} c_{r e d^{*}} \sqrt{\frac{F V}{R T D_{r e d}}} \frac{d \bar{a}(X, s)}{d X}\right|_{x=0}
$$

From Eqn. (A8),we get

$$
\left.\frac{\partial \bar{a}(X, s)}{\partial X}\right|_{X=0}=c_{1} \sqrt{s} e^{\sqrt{s \gamma}}
$$

Using Eqns. (A10) and (A11)

$$
\frac{i(s)}{D_{\text {red }} C_{\text {red }} * n F A \sqrt{\frac{F V}{R T D_{\text {red }}}}}=\left.\frac{d \bar{a}(X, s)}{d X}\right|_{x=0}=c_{1} \sqrt{s} e^{\sqrt{s \gamma}}
$$

The above equation can be written as

$$
\frac{i(s)}{m}=\left.\frac{d \bar{a}(x, s)}{d X}\right|_{x=0}=c_{1} \sqrt{s} e^{\sqrt{s \gamma}}
$$

Where

$$
m=\frac{1}{D_{\text {red }} C_{\text {red }}{ }^{*} n F A \sqrt{\frac{F V}{R T D_{\text {red }}}}}
$$

Now the constant $c_{l}$ becomes

$$
c_{1}=\frac{i(s)}{m \sqrt{s} e^{\sqrt{s \gamma}}}
$$

Using this constant the Eqn. (A8) beomes

$\bar{a}(0, s)=\frac{i(s)}{m \sqrt{s}}\left[2 e^{-\sqrt{s \gamma}}-1\right]+\frac{1}{s}=\frac{2 i(s)}{m} e^{-\sqrt{s \gamma}}-\frac{i(s)}{m \sqrt{s}}+\frac{1}{s}$

Taking laplace inverse on bothsideswe get

$$
a(0, \tau)=1-\frac{1}{m \sqrt{\pi}}\left\{\int_{0}^{\tau} \frac{i(\tau)}{\sqrt{t-\tau}}\left[1-2 \exp \left(-\gamma^{2} / 4(t-\tau)\right] d \tau\right)\right\}
$$

Which is the equation (17) in the text.

\section{References}

[1] Group Southampton Electrochemistry, Instrumental methods in electrochemistry (Ellis Horwood, Chichester, (2001)).

[2] D. Britz and B. Kastening, "On the electrochemical observation of a second-order decay of radicals generated by flash photolysis or pulse radiolysis," Journal of Electroanalytical Chemistry, 56(1), 73-90 (1974).

[3] F. Lantelme and E. Cherrat, "Application of cyclic voltammetry to the study of and metalliding processes," Journal of Electroanalytical Chemistry 244(1-2), 61-68(1988).

[4] N. White and F. Lawson, "Potential sweep voltammetry of metal deposition and dissolution Part I. Theoretical analysis," Journal of Electroanalytical Chemistry 25(3), 409-419 (1970).

[5] R. S. Nicholson and Irving Shain, "Theory of stationary electrode polarography. single scan and cyclic methods applied to reversible, irreversible, and kinetic systems," Anal. Chem. Analytical Chemistry 36(4), 706-723 (1964).

[6] Allen J. Bard and Larry R. Faulkner, Electrochemical methods : Fundamentals and Applications (Wiley, New York, 2001).

[7] L. Bortels, B. Van Den Bossche, and J. Deconinck, "Analytical solution for the steady-state and migration. Application to the identification of Butler-Volmer electrode reaction parameters," Journal of electroanalytical chemistry 422(1/2), 161-167 (1997).

[8] A. Molina, E. Torralba, C. Serna, and J. A. Ortuno, "Analytical solution for the facilitated ion transfer at the interface between two immiscible electrolyte solutions via successive complexation reactions in any voltammetric technique: Application to square wave voltammetry and cyclic voltammetry," Electrochim Acta Electrochimica Acta 106, 244-257 (2013). 
[9] TalivaldisBerzins and Paul Delahay, "Oscillographicpolarographic waves for the reversible deposition of metals on solid electrodes," Journal of the American Chemical Society 75(3), 555-559 (1953).

[10] Jan C. Myland and Keith B. Oldham, "Quasireversible cyclic voltammetry of a surface confined redox system: a mathematical treatment," Electrochemistry Communications 7(3), 282-287 (2005).

[11] Keith B. Oldham and Jan C. Myland, "Modelling cyclic voltammetry without digital simulation," Electrochimica Acta Electrochimica Acta 56(28), 10612-10625 (2011).

[12] A. Eswari and L. Rajendran, "Mathematical modeling of cyclic voltammetry for EC reaction," Russian Journal of Electrochemistry 47(2), 181-190 (2011).

[13] A. Eswari and L. Rajendran, "Mathematical modeling of cyclic voltammetry for EC2 reaction," Russian Journal of Electrochemistry 47(2), 191-199 (2011).
[14] Adib J. Samin, and Jinsuo Zhang, "Analytical solutions of the plannar cyclic voltammetry process for two soluable species with equal diffusivities and fast electron transfer using the method of eigen function expansion", AIP Advances, 5 087141,(2015).

[15] Tanja R. Vidakovic-Koch,Vladimir V. Panic, Milan AndriMenkaPetkovska, Kai Sundmacher, "Nonlinear frequency response analysis of the ferrocyanideoxidation kinetics. part i. a theoretical analysis", J. Phys. Chem. C 2011, $115,17341-17351$.

[16] G. Moutiers, M. Cassir, J. Devynck, "A thermodynamic, voltammetric and convolution potential sweep characterization", J. Electronal. Chem. 324(1992) 175-189. 